\title{
Evaluation of patient transfers from peripheral hospitals to a District General Hospital
}

\author{
Abeysekara $\mathrm{CM}^{1}$, Hewageegana $\mathrm{NR}^{2}$, Dharmarathtne $\mathrm{SD}^{3}$ \\ ${ }^{1}$ Deputy Director,District General Hospital, Matara. \\ ${ }^{2}$ Senior Lecturer, Department of Medicine, University of Peradeniya. \\ ${ }^{3}$ Associate Professor, Department of Community Medicine, University of Peradeniya.
}

\begin{abstract}
Introduction: Despite Sri Lanka's remarkable success of achieving higher health status, there are many areas in the system which need immediate and priority attention to further improve the provision of healthcare. Patient transfer procedure is one such area.

Objectives: This study was undertaken to evaluate the patient transfers from peripheral hospitals to District General Hospital (DGH), Matara and to make recommendations to improve the process.

Materials and Methods: A descriptive cross-sectional study was carried out using three study instruments. A questionnaire was administered to a convenience sample of 427 transferred patients and medical officers who attended them at the Primary Care Unit (PCU). Two checklists were used to assess the completeness of the components of the transfer process. Data were triangulated and analyzed regarding proportions (quantitative data) and associations (using chi-square test).

Results: Involvement of the patient/guardian to the decision-making process was inadequate as $62.5 \%$ of the patients/guardians were not aware of the reason for the transfer. Pre-transfer communication was not done in $90.9 \%$ occasions. Majority of transferred patients (74.5\%) were accompanied by staff attendants irrespective of the nature of the transfer. There were many lapses in the documentation of the transfer form.
\end{abstract}

Discussion: Guidelines for inter-hospital patient transfers need to be revised. The transfer form used at present need to be redesigned.

\section{Introduction}

Sri Lankan government curative health system consists of network of health care institutions organized in to three tiers of primary, secondary and tertiary levels. In this hierarchical pyramidal network, higher level facilities act as referral centers for lower level facilities. ${ }^{1}$

Indications for Inter-institutional transfers of patients include provision of specialty or subspecialty care, non-availability of medical treatment, diagnostic facilities or Intensive Care Unit (ICU) beds and continuation of medical care at a lower tier institution nearest to the patient residence. Established primary, secondary and tertiary levels of hierarchical structure provide ideal background for flow of patients in both ways. But many improper transfers happen due to the technical, competency and organizational flaws in the system resulting wastage of health care resources, burden to the health care 
staff, impairment of quality of health care provision, potential risk to the patients, patient dissatisfaction and loss of productivity to the society. ${ }^{2}$

At present inter institutional transfers within the government health system are conducted according to the circular issued by the ministry of health in 2002. ${ }^{3}$ This circular was not updated for the last 15 years though the country experienced significant improvement of island wide health care facilities and services. Evidence was not available in the literature regarding any studies or audits conducted to evaluate the adherence to the said circular when transferring patients between healthcare institutions.

This study was undertaken with the view of evaluating the patient transfers within the government health sector and making recommendations to improve the process.

\section{METHODS}

A descriptive cross-sectional studywas carried out at the PCU of the District General Hospital, Matara. A convenient sample of 427 was selected. All the transferred patients from peripheral hospitals to district General Hospital, Matara were taken as the study population. Data collection was done from 30th April 2018 to 14th May 2018.

One questionnaire and two check lists were used for the study as instruments.Questionnaire was used to collect socio demographic profile data of transferred patients and to assess the completeness of the components related to patient transfer process. Check list 1 was designed according to the items mentioned in the transfer form. Check list 2 was designed considering the information available in the Bed Head Ticket.

Data was analyzed using SPSS 22 version software.Ethical clearance was obtained from the ethics review committee of the Post Graduate Institute of Medicine, University of Colombo.

\section{RESULTS}

Out of 427 transferred patients, $50.6 \%(n=216)$ were male. Age of the sampled patients was normally distributed with mean age of 39.17 years and standard deviation of 26.24. Their age distribution is shown in Table 1.

Table 1 - Age distribution of transferredpatients

\begin{tabular}{|l|l|l|}
\hline Age (years) & Frequency & Percentage (\%) \\
\hline $0-1$ & 12 & 2.8 \\
$2-5$ & 34 & 8 \\
$6-14$ & 57 & 13.3 \\
$15-29$ & 68 & 15.9 \\
$30-44$ & 79 & 18.5 \\
$45-59$ & 63 & 14.8 \\
$60-74$ & 60 & 14.1 \\
$75-89$ & 48 & 11.2 \\
$>90$ & 6 & 1.4 \\
\hline Total & 427 & 100 \\
\hline
\end{tabular}

After excluding the pediatric age group, 255 (78.7\%) out of 324 patients were married.Majority of transferred patients $(86.7 \%, n=370)$ were Sinhalese while $9.6 \%(n=41)$ were Tamils and $3.7 \%(n=16)$ were Muslims. As expected, the distribution of transferred patient's religions showed similar pattern to the ethnicity with majority (78.5\%) being Buddhists. 
Most of the patients in the sample $(39.6 \%, n=151)$ were educated up to ordinary level while a considerable number $(7.6 \%, \mathrm{n}=29)$ had not even attended school. Their level of education is shown in figure 2 .

Table 2 - Level of education of transferred patients

\begin{tabular}{|l|l|l|}
\hline Level of education* & Frequency & Percentage (\%) \\
\hline No schooling & 29 & 7.6 \\
Grade 1-4 & 62 & 16.3 \\
Grade 5-9 & 81 & 21.2 \\
Ordinary level & 151 & 39.6 \\
Advance level & 52 & 13.6 \\
Graduate & 6 & 1.5 \\
Postgraduate & 0 & 0 \\
\hline
\end{tabular}

$45.2 \%$ of the patients were unemployed.

Most of the patients were transferred to DGH Matara from Base Hospital,Deniyaya (18.3\%, $n=78)$, District Hospital,Akuressa $(14.8 \%, n=63)$, Base Hospital Kamburupitiya (11.5\%, $n=49)$, Base Hospital Tangalle $(9.4 \%, n=40)$ and Base Hospital Walasmulla $(8.2 \%, n=35)$.

Table 3: Distribution of transferred patients by transfer originated hospital

\begin{tabular}{|l|l|l|}
\hline Hospital & Frequency & Percentage (\%) \\
\hline Deniyaya & 78 & 18.3 \\
\hline Akuressa & 63 & 14.8 \\
\hline Kamburupitiya & 49 & 11.5 \\
\hline Tangalle & 40 & 9.4 \\
\hline Walasmulla & 35 & 8.2 \\
\hline Morawaka & 33 & 7.7 \\
\hline Dikwella & 28 & 6.6 \\
\hline Kamburugamuwa & 27 & 6.3 \\
\hline Weligama & 20 & 4.7 \\
\hline Talalla & 12 & 2.8 \\
\hline Urugamuwa & 9 & 2.1 \\
\hline Hambantota & 8 & 1.9 \\
\hline Kirinda & 6 & 1.4 \\
\hline Gangodagama & 6 & 1.4 \\
\hline Urubokka & 4 & .9 \\
\hline Narawelpita & 3 & 7 \\
\hline Kirama & 2 & 5 \\
\hline Beliatta & 2 & 5 \\
\hline Thissamaharamaya & 2 & 5 \\
\hline Total & 427 & 100.0 \\
\hline
\end{tabular}

The mean distance to the peripheral hospital from the residence of the patients was $6.34 \mathrm{~km}$. Almost one third of patients $(29.5 \%, \mathrm{n}=126)$ were living within three kilometers of the admitted hospital. The mean distance to DGH Matara from the patients' residence was $41.1 \mathrm{~km}$. Almost half of the patients 
$(49.9 \%, \mathrm{n}=213)$ were residing at $20 \mathrm{~km}$ to $39 \mathrm{~km}$ away from the DGH Matarawhile $68(15.9 \%)$ patients were at $80 \mathrm{~km}$ to $99 \mathrm{~km}$ distance from the DGH Matara.

Obstetric conditions $(16.4 \%, n=70)$ accounted for the highest number of the transfers from peripheral hospitals. Traumatic injuries and poisoning $(15 \%, n=64)$ accounted for next highest number of transfers. Cardiovascular and respiratory disorders accounted for $12.6 \%(n=54)$ and $11.2 \%(n=48)$ of transfers respectively.

Table 4: Distribution of sample by the reason for transfer

\begin{tabular}{|l|l|l|}
\hline Reason & Frequency & Percentage (\%) \\
\hline Pregnancy related & 70 & 16.4 \\
Injury \& poisoning & 64 & 15.0 \\
Cardiovascular Disorder & 54 & 12.6 \\
Respiratory Disease & 48 & 11.2 \\
Symptoms, signs \&abno* & 42 & 9.8 \\
Infection & 36 & 8.4 \\
Ear Disease & 24 & 5.6 \\
Genitourinary Disease & 23 & 5.4 \\
Gastrointestinal Disease & 16 & 3.7 \\
Neurological Disease & 10 & 2.3 \\
Skin Disease & 8 & 1.9 \\
Musculoskeletal Disorder & 8 & 1.9 \\
Perinatal Conditions & 8 & 1.9 \\
Blood Related Disorders & 4 & 0.9 \\
Endocrine/Nutrition/Metabolic & 4 & 0.9 \\
Eye Disease & 4 & 0.9 \\
Neoplasm & 2 & 0.5 \\
Psychiatric Disorders & 2 & 0.5 \\
\hline Total & 427 & 100 \\
\hline
\end{tabular}

*Abnormal clinical findings

In most of the occasions $(38.4 \% \mathrm{n}=164)$ designation of the transferring officer was not mentioned.In one third of occasions $(33.3 \%, n=142)$ medical officer was the transferring officer. In one fourth of occasions $(25.2 \%, n=110)$ relevant consultant's designation was mentioned as the transferring officer. Majority of patients/guardians $(73.3 \%, n=313)$ were informed either by the consultant or a medical officer regarding the transfer. In 112 (26.2\%) instances patients/guardians were informed by nursing officers. Majority of patients or their guardians $(62.5 \%, \mathrm{n}=267)$ were aware of the reason for their transfer to DGH Matara. In $53.6 \%$ of transfers $(n=229)$, neither the patient nor the guardian was informed that they were consented for transferring, even though they did put their signature on Bed Head Tickets. (But for majority of transfers $(53.6 \%, n=229)$ either the patient or the guardian was not aware of giving consent prior to transfer even though they should have put their signatures on Bed Head Tickets.)

Out of 427 transfers $58.8 \%(n=251)$ were labeled as "urgent transfers" by medical officers in the peripheral hospitals while $41.2 \% \quad(n=176)$ belonged to non-urgent category. Pre-transfer communications were done with DGH Matara only regarding $8.7 \%(n=37)$ transfers. Even for 251 urgent transfers, pre-transfer communication was done only in $11.1 \%(n=28)$ occasions. 
A significant majority of urgent transfers $(80.4 \%, n=202)$ were accompanied either by an attendant or a labourer only. All the non-urgent transfers excluding pregnancy related cases were accompanied either by an attendant $(82.3, n=145)$ or by a laborer $(7.9 \%, n=14)$

Transfer form was the only document handed over with the patient on admission to DGH Matara in 93.4\% ( $n=399)$ occasions.

In all transfer forms, name of the DGH Matara was written either accurately $(96.2 \%, \mathrm{n}=411)$ or inaccurately $(3.7 \%, n=16)$. Patent's name and gender was not mentioned in $1.4 \%(n=6)$ and $4.2 \%(n=18)$ of transfer forms respectively. Name and address of the guardian was not written $3.3 \%(n=14)$ and $6.1 \%$ $(n=26)$ of occasions. In more than half of the transfer forms ethnicity $(64.6 \%, n=276)$, religion $(71.2 \%$, $n=304)$ and address $(65.1 \%, n=278)$ of the patient were not mentioned.

In $239(56 \%)$ instances back of the transfer form was used to mention added information about the patient.

\section{DISCUSSION}

Overall this study revealed some vital findings which would be useful for the planning of health care service in the future.

Sex ratio among the transferred patients was almost equal. Reproductive age group (15 to 44 years) accounted for $34.4 \%$ of transfers reflecting that the commonest reason for the transfers were obstetric causes. One fourth of the patients (26.7\%) belonged to the senior citizen group ( $>60$ years). Majority of the transferred patients were Sinhala Buddhists. These findings were compatible with existing demographic pattern of the region.

Further analysis of age and gender characteristics of the sample together revealed that females were predominant below 45 years age group and males were predominant above 45 -year age group. 70 cases of pregnant patients who belong to reproductive age group would have contributed for the observed difference. Notably higher number of injuries and poisoning, respiratory diseases and Infections were reported among males in the sampled population.

Further analysis of data revealed that most Tamil patients (25 out of 41 or $60.9 \%$ ) were females and transferred from Base Hospital Deniyaya, where tea plantation is widespread than the other areas of the district. Of them 15 (60\%) were transferred due to pregnancy related reasons. These findings need to be further studied as they indicate the quality of obstetric care received by this relatively vulnerable community.

There were only 6 graduates (1.4\%) and none of the patients had post graduate qualifications.

Females were the dominant majority among unemployed $(57.7 \%)$ while $66.2 \%$ were males among the employed category. This difference could be due to the traditional attitudes prevalent in the rural population regarding the female employment.

BH Deniyaya (18.3\%), DH Akuressa (14.8\%), BH Kamburupitiya (11.5\%), BH Tangalle (9.4\%) and BH Walasmulla (8.2\%) together accounted for the majority (62.1\%) of transfers to Matara hospital. Except DH Akuressa other hospitals belonged to secondary care category. Further studies to identify the 
underlying reasons for indications of transfers from secondary level to tertiary level would help to upgrade the facilities at Base Hospitals and to avoid unnecessary transfers.

The mean distance from the residence of patients to admitted hospital was $6.34 \mathrm{~km}$. Two thirds of the patients $(67.4 \%)$ were residing within seven kilometers of distance from the respective peripheral hospitals. This fact highlights both the easy accessibility to the health care services at rural areas and the satisfactory level of utilization of available services at primary and secondary care levels.

The mean distance from the patients' residence to DGH Matara was $41.1 \mathrm{~km}$. Despite the improvement of the road network transport facilities recently, still it would take more than one hour for majority of patients' relatives to reach DGH Matara. In addition, they must bear considerable amount of out of pocket expenditure to visit the patient. Medical officers working at peripheries need to keep these facts in mind when making decisions to transfer patients.

It is important to note that in most of the occasions (38.4\%), the designation of the transferring officer was not mentioned. Out of 239 transfers sent from secondary or tertiary care hospitals to DGH Matara, in $37.6 \%$ occasions medical officers were the authorizing person for the transfer even though consultants were present in these hospitals raising the possibility that they were either on leave or there concurrence obtained but not documented.

According to the literature, the best practice is that the decision to transfer should be made by clinicians with the involvement of the patient/guardian. ${ }^{4}$ But the existing practice in here appears to be different as considerable proportion of patients (26.2\%) were informed regarding the transfer to DGH Matara by the nursing officers. Less patient's involvement regarding decision making process was further confirmed by the fact that $37.5 \%$ of the transferred patients were not aware about the reason for their transfer to higher institution.

Enough attention was not paid to the patient's or guardian's consent as in majority of transfers (53.6\%) either patient or bystander was not aware that their consent was taken prior to transfer even though they must have put their signatures on Bed Head Tickets.

Analyzed results revealed that pre-transfer communicationswere done only regarding $8.7 \%$ transfers. The results signify the lack of attention for one of the most important components of the patient transfer process by the implementers of patient transfers. The possible reasons would be the lack of emphasis given in the existing circular for this component.

It is important to note that 202 urgent transfers (80.5\%) were accompanied by either an attendant or a laborer alone. Lack of the staff at peripheral level may have contributed to this. Selection of the accompanying personnel regarding the transferring patient's needs to be further elaborated in existing guidelines. Creation of separate paramedical carder as in many other countries needs to be considered since they can be utilized as accompanying personnel in many occasions. ${ }^{5}$

Results of the study revealed that the transfer form was the only document handed over with the patient on admission to the DGH Matara in $93.4 \%$ occasions. A medical officer or a nursing officer was involved in handing over of only 33 patients (7.7\%). Patient handing over process should be elaborated in the existing circular. 
Accurate documentation was another important component of the transfer process. There were many lapses regarding the documentation of Patient' ethnicity, religion and address, registered number and ward number of peripheral hospital and the designation of the transferring officer in the transfer form. Transfer time was mentioned in the transfer forms in $80.1 \%$ occasions. Still this figure could be considered as satisfactory since Health 946 form didn't contain a defined space to mention the time. The reason for incomplete documentation could be that the medical officersand house officers failed to pay enough attention with this regard due to heavy workload. The belief of the transferring officer that such information may not be relevant for patient management is another reason for those lapses. Appointment of ward clerks would relive medical officers from the additional burden of documentation of non-clinical entries in the transfer form and would allow them to concentrate more on the clinical work. Such a cadre is available in many health systems to assist medical staff with clerical tasks. ${ }^{6}$ This is especially important in resource deficient set ups such as peripheral hospitals.

In majority of instances (56\%) back of the transfer form was utilized to mention information about the transfer. Utilization of the opposite side of the form indicates that the space available in the front of the form for documentation is inadequate. Special attention needs to be paid in this regard when designing a new transfer form.

Patient transfer process involves both transfer originating hospital and transfer receiving hospital. The study setting was limited to DGH Matara, the receiving end of the process, due to the difficulties of visiting number of peripheral hospitals in the region. If some components of the process such as pretransfer communication and pre-transfer stabilization were assessed at the origin of transfers, the study would be more complete.

\section{Recommendations}

Guidelines for inter-hospital patient transfers need to be revised as the relevant circular was issued way back in 2002. The transfer form used at present need to be redesigned with inclusion of certain items such as the time of the transfer, accompanying staff, pre-transfer stabilization etc. Only the essential socio-demographic data of the patient must be included to avoid added burden to the transferring officer. An adequate space needs to be given for documentation of clinical material. A separate areaneeds to be allocated to mention additional information or retransfer information about the patient when relevant.

Introduction of departure check list would prevent many short comings.

A separate information management system needs to be established to implement both inter-hospitals as well as intra-hospital patient transfers effectively. Up to date interfacility communication system need to be introduced. Appointment of ward clerk to assist clinical staff would improve documentation of the transfer form.

Creation of a separate cadre of paramedics needs to be considered as they can be utilized as accompanying personnel in majority of transfers.

Facilities at secondary care level should be upgraded to minimize the number of transfers from base hospitals to tertiary care hospital like DGH Matara.Regular audits and periodic reviews must be conducted regarding the transfer process at all levels of care. 


\section{References:}

1. Huntington, D. and Hort, K. Public hospital governance in Asia and the Pacific. Geneva: World Health Organization; 2015. Available from:

http://www.searo.who.int/entity/asia_pacific_observatory/publications/country_comparative_studi es/ccs_public_hospital_governance/en/ [Accessed 14 October 2019].

2. Sellahewa K, Lamabadusuriya S, Edirisinghe E. Transfers to a general medical ward at National Hospital of Sri Lanka: a descriptive study. Galle Medical Journal. 2009;14(1):5.

3. Department of Health Services. Transfer of patients from one institution to another. Colombo: Ministry of Health; 2002. Available from:

http://www.health.gov.lk/CMS/cmsmoh1/upload/english/01-09-2002-eng.pdf [Accessed 14 October 2019].

4. Kulshrestha A, Singh J. Inter-hospital and intra-hospital patient transfer: Recent concepts. Indian Journal of Anaesthesia. 2016;60(7):451.

5. The Association of Anaesthetists of Great Britain and Ireland. AAGBI safety guideline: Interhospital transfer. Available from: https://www.aagbi.org/sites/default/files/interhospital09.pdf [Accessed 15 Oct. 2018].

6. Health Careers. Clerk. Available from: https://www.healthcareers.nhs.uk/explore-roles/widerhealthcare-team/roles-wider-healthcare-team/administration/clerk [Accessed 14 October 2019]. 